\title{
Determinación del historial de exposiciones en la epidemiolgía ocupacional
}

\author{
Maria Teresa Espinosa, ${ }^{1}$ Timo Partanen, ${ }^{2}$ Marion Piñeros, ${ }^{3}$ Jorge Chaves, ${ }^{4}$ \\ Héctor Posso, ${ }^{3}$ Patricia Monge, ${ }^{2}$ Luís Blanco ${ }^{5}$ y Catharina Wesseling ${ }^{2}$
}

Forma de citar

Espinosa MT, Partanen T, Piñeros M, Chaves J, Posso H, Monje P, Blanco L, Wesseling C. Determinación del historial de exposiciones en la epidemiología ocupacional. Rev Panam Salud Publica. 2005; 18(3):187-96.

RESUMEN La validez de los indicadores de exposición es una condición necesaria en epidemiología si se han de obtener resultados válidos en la medición de los riesgos asociados con la exposición a agentes nocivos en el entorno laboral. Sin embargo, llevar a cabo la validación de estos indicadores de exposiciones pasadas no es tarea fácil.

Debido a la falta de mediciones de referencia en el ámbito de la higiene industrial y de concentraciones representativas de bioindicadores que reflejen las exposiciones pasadas, el método de los autoinformes se ha utilizado para recoger datos de exposición indirectos. No obstante, los datos acerca de agentes nocivos específicos son a menudo deficientes y deben completarse con otros sobre los factores condicionantes de la exposición. La validez de los autoinformes mejora cuando se utilizan listas de verificación e iconos ilustrativos, mientras que la calidad de la información sobre las exposiciones personales mejora cuando se incorporan datos secundarios acerca de las exposiciones y de los factores que las condicionan o determinan.

La exposición se puede determinar mediante matrices de exposición, evaluación por expertos y modelos de exposición, integrando datos primarios y secundarios acerca de las exposiciones y sus factores condicionantes. Las matrices contienen datos agrupados y, por consiguiente, pueden llevar a errores a la hora de clasificar las exposiciones individuales e introducir sesgos en la estimación de los riesgos.

La evaluación por expertos es probablemente el método con el índice de validez más alto, pero puede entrañar costos muy altos en el caso de estudios de cierta magnitud. Otra posibilidad con buenas perspectivas es la de utilizar un modelo formal para evaluar las exposiciones patentes y mejorarlo mediante la evaluación por expertos en situaciones en las cuales los resultados del modelo parezcan alejarse de la realidad.

Palabras clave Exposición ocupacional, epidemiología/métodos, medición de riesgo, riesgos laborales.

1 Posgrado de Salud Ocupacional, Universidad El Bosque, Santa Fe de Bogotá, Colombia. La correspondencia debe dirigirse a: María Teresa Espinosa, Profesora Asociada, Directora del Posgrado de Salud Ocupacional, Universidad El Bosque, Calle 134 \# 13-83, Santa Fe de Bogotá, Colombia. Tel: (571) 6489076; fax: (571) 6489040. Correo-e: espinosamaria@unbosque.edu.co; materespinosa@ yahoo.com
2 Instituto Regional de Estudios en Sustancias Tóxicas, Universidad Nacional, Heredia, Costa Rica. Instituto Nacional de Cancerología, Santa Fe de Bogotá, Colombia.

4 Instituto Tecnológico de Costa Rica, San José, Costa Rica.

5 Programa de Salud Ocupacional y Ambiental, Universidad Nacional Autónoma, León, Nicaragua.
Los métodos que permiten determinar el historial de exposición individual están adquiriendo cada vez más importancia en la epidemiología ocupacional para la cuantificación fiable de asociaciones entre las exposiciones reales y los parámetros de salud y en- 
fermedad, usando tantos indicadores binarios (no-sí) de la exposición como indicadores de la relación entre dosis y efecto. La estimación de esta relación deberá estar dada en términos de intensidad, duración, dosis acumulada, temporalidad de la exposición, tipo de actividad económica, oficio, zona geográfica, período de latencia y otros factores condicionantes del riesgo de enfermar. En el campo de la epidemiología ocupacional, para el estudio de enfermedades con largos períodos de latencia es necesario reconstruir la exposición en el pasado con el fin de poder calcular con mayor precisión el grado de asociación y lograr mayor sensibilidad a la hora de detectar relaciones de causalidad.

Se distinguen por lo menos tres grandes grupos de métodos para determinar de la exposición pasada, entre ellos las matrices de exposición laboral para la población en general o para sectores específicos, las evaluaciones a cargo de expertos, y los modelos de exposición, cada uno con sus ventajas y limitaciones. La identificación cualitativa de exposiciones y riesgos está dando lugar a su evaluación cuantitativa y semicuantitativa $(1,2)$. La validez de los indicadores cuantitativos y semicuantitativos de exposición es condición necesaria para obtener resultados fidedignos al medir los riesgos asociados con exposiciones a agentes nocivos en el ambiente laboral. Un aspecto crucial de esta cuestión es el hecho de que, desde el punto de vista etiológico, es muy difícil saber en qué medida los indicadores empíricos de exposición corresponden a la exposición real, ya que habitualmente no hay datos acerca de las exposiciones reales durante los períodos de mayor exposición. Así ha sucedido especialmente en el pasado con exposiciones que han desempeñado un papel condicionante en la evolución de enfermedades con períodos prolongados de latencia, como la mayor parte de los cánceres. Los errores en los indicadores empíricos pueden considerarse desviaciones de los valores verdaderos, si estos corresponden a una variable continua, o como proporciones de resultados negativos falsos (poca sen- sibilidad) y de resultados positivos falsos (poca especificidad), si se trata de variables categóricas.

En la presente revisión, el término "determinación de la exposición" traduce el concepto de exposure assessment para evitar confundirlo con la evaluación cuantitativa de la exposición. Esta determinación de la exposición puede desglosarse a su vez en dos variantes: la determinación de la exposición actual y la determinación del historial de exposición, es decir, de la exposición que ha habido en el pasado. A esta última se hace referencia en el presente artículo, en el que se aborda la determinación de la causalidad con fines epidemiológicos.

La determinación de la exposición en la epidemiología ocupacional moderna se refiere a la conversión de datos primarios y secundarios acerca de las exposiciones y de sus factores condicionantes en indicadores derivados de la exposición individual. La conversión se realiza con algoritmos específicos que representan grados variables de formalidad y complejidad. Obviamente, con ello se persigue el objetivo de obtener indicadores de la exposición personal -la exposición acumulada, el tiempo de exposición, y otros factores condicionantes del riesgo de enfermar-, a fin de poder analizar el riesgo individual.

El estudio de casos y testigos representa una situación prototípica. En primer lugar, se buscan indicadores de exposiciones pasadas a agentes predefinidos, preferiblemente en ventanas de tiempo relacionadas con el comienzo de la exposición, el diagnóstico de los casos y otros momentos clave. En teoría, el indicador básico de exposición es la intensidad de la exposición como función continua del tiempo. A partir de esta función básica se pueden determinar todos los indicadores importantes: grado de exposición promedio ocupacional, duraciones, frecuencias, exposiciones acumuladas y la ocurrencia e intensidad de los picos de exposición. En la práctica, los datos disponibles permiten abordar el estudio de estos indicadores con mayor o menor garantía de fiabilidad. Un área importante, aunque menos desarro- llada, es la determinación de exposiciones cutáneas, cuya evaluación plantea problemas particulares (3).

En situaciones en las que se dispone de un indicador directo de la exposición pasada, como sería un bioindicador que dura en el organismo largo tiempo, no se necesitará ninguna conversión; sin embargo, en la práctica no es frecuente disponer de datos de exposición directa, sino más bien de datos acerca de los factores condicionantes de la exposición.

En este artículo se describirán las fuentes primarias y secundarias de datos de exposición y sus factores condicionantes, así como los métodos que pueden usarse para determinar las exposiciones anteriores a partir de datos primarios y secundarios.

\section{Datos de exposición y factores condicionantes de la exposición}

El concepto de factor condicionante de la exposición se refiere a todo factor que ejerce alguna influencia sobre la exposición (cuadro 1). Los factores condicionantes pueden clasificarse en relación con el agente (p. ej., características fisicoquímicas, persistencia en el organismo, bioacumulación y toxicidad, entre otras); con las condiciones ambientales (p. ej., temperatura y ventilación); con el lugar de trabajo (tipo de proceso de producción y de tecnología, entre otros); con la organización del trabajo (frecuencia y duración de la estancia en cada puesto de trabajo), y con las características del trabajador (p. ej., obesidad, consumo de alcohol y de tabaco, estado de salud, edad, sexo y raza, entre otros) (2). Los datos correspondientes a los factores condicionantes de la exposición son parámetros importantes de los que pueden derivarse los indicadores de exposición, sobre todo cuando no existen datos de exposición directos.

Un indicador derivado o un dato derivado de exposición se refiere a una variable de exposición personal, de carácter secundario, que se encuentra durante el proceso de la determinación de la exposición. El riesgo alude estrictamente a la probabilidad de producir un efecto adverso para la sa- 


\section{CUADRO 1. Ejemplos de factores condicionantes de exposición}

\begin{tabular}{|c|c|c|}
\hline Clase de factor condicionante & Ejemplo de factor condicionante & Ejemplo de exposición determinada \\
\hline Ocupación & Clasificación internacional de Ocupaciones & Múltiples exposiciones laborales \\
\hline Tarea, puesto, proceso de producción & Tarea agrícola & Nivel de exposición a plaguicidas específicos \\
\hline Frecuencia de la tarea, proceso o aplicación & $\begin{array}{l}\text { Frecuencia de la aplicación de glifosato durante } \\
\text { un año }\end{array}$ & Exposición acumulada a glifosato y su nivel \\
\hline Duración de la tarea o proceso & Duración de la aplicación de glifosato & Exposición acumulada a glifosato \\
\hline Equipo & Equipo de aplicación de plaguicida(s) & Nivel de exposición a plaguicida(s) \\
\hline Maquinaria & Velocidad de la máquina de lijado del ebanista & Nivel de exposición al polvo de madera \\
\hline Maquinaria & Temperatura en la presión del aserrín comprimido & Nivel de exposición al formaldehído \\
\hline Cantidad de material consumido & Cantidad del tolueno usado en una prensa & Nivel de exposición al tolueno \\
\hline Automatización & $\begin{array}{l}\text { Automatización de la clasificación de calidad del } \\
\text { contrachapado (triplex) }\end{array}$ & Menos trabajadores expuestos \\
\hline $\begin{array}{l}\text { Controles del proceso, control técnico y } \\
\text { administrativo }\end{array}$ & $\begin{array}{l}\text { Extracción de polvo de madera en un taller de } \\
\text { ebanista con campanas de extracción. } \\
\text { Rotación de puestos. }\end{array}$ & Nivel de exposición al polvo de madera \\
\hline Protección personal & $\begin{array}{l}\text { Uso de pantalones largos durante la aplicación } \\
\text { de plaguicidas }\end{array}$ & Nivel de exposición cutánea al plaguicida(s) \\
\hline Vía de ingreso & Piel & Nivel de exposición \\
\hline Área de superficie de la piel en contacto & $\begin{array}{l}\text { Porcentaje de la superficie cutánea en contacto } \\
\text { con cemento }\end{array}$ & Absorción dérmica de agente(s) \\
\hline
\end{tabular}

Nota: Véase la referencia 35.

lud. En este artículo se emplea la evaluación o la determinación del riesgo solamente a modo de ejemplo, y se centra la atención en la evaluación o estimación de las exposiciones (que podrán entenderse como factores condicionantes del riesgo).

Datos primarios. Los datos primarios, que pueden corresponder a las exposiciones mismas o a sus factores condicionantes, pueden proceder de diferentes fuentes: datos administrativos (empresas, gobiernos, sociedades gremiales), autoinformes o entrevistas a familiares sobre las condiciones de trabajo, o resultados de evaluaciones personales o de determinación de bioindicadores específicos en poblaciones objeto de estudio.

Datos administrativos. Entre las fuentes de estos datos primarios cabe mencionar los archivos pertenecientes al censo; a registros empresariales o gremiales; a servicios médicos; a sistemas de vigilancia de salud pública o de salud ocu- pacional (por agente nocivo o por efecto en la salud) y de sucesos centinelas; a sistemas de registro de trastornos $y$ enfermedades en empresas y lugares de trabajo; a sistemas de seguros; y a sindicatos. Muchas de estas fuentes se establecieron con fines administrativos o para otros propósitos diferentes a la determinación de la exposición. Por eso no son modificables, lo cual limita su uso para estudios de determinación de la exposición. En algunos casos, ciertos tipos de variables se pueden reclasificar (se puede, por ejemplo, agrupar en nuevas categorías los sectores económicos u ocupaciones).

No siempre se dispone de los datos administrativos pertinentes. Los datos disponibles pueden tener poca validez, dado que en un origen se recolectaron por motivos distintos.

Historiales ocupacionales obtenidos por autoinformes y entrevistas. En contraposición con estos datos administrativos, los investigadores han ideado métodos para recolectar datos primarios ad hoc, tales como cuestionarios de autoinformes e informes de familiares, amigos o colegas, sobre datos retrospectivos de empleo, puestos de trabajo $\mathrm{y}$ tareas, productos y servicios $\mathrm{y}$, hasta cierto punto, también sobre exposiciones a agentes específicos y distintas clases de sustancias. Se han elaborado diferentes procedimientos, algunos sencillos y otros bastante extensos pero que integran mucha información, a fin de realizar entrevistas para recoger los historiales ocupacionales. Dichas entrevistas pueden contener módulos específicos de acuerdo con el sector industrial o la ocupación en cuestión y listas de control de tareas y agentes; pueden efectuarse como mínimo en ocasiones diferentes con el fin de verificar o complementar la información suministrada y pueden llevarse a cabo con el uso de una computadora, previa selección y preparación cuidadosa de los encuestadores (4-9).

La factibilidad de usar autoinformes obtenidos mediante entrevistas personales se ve limitada por el número de 
sujetos. En casi todos los países, especialmente en los países en desarrollo, las encuestas postales nos son viables, y las entrevistas telefónicas solo se pueden realizar en algunos países desarrollados.

La validez de datos directos acerca de la exposición a agentes específicos obtenidos mediante autoinformes es sumamente variable. En diferentes estudios se ha informado de coeficientes de determinación (coeficientes de correlación al cuadrado, $r^{2}$ ) de 0,03 a 0,7 para diferentes agentes, frente a datos de exposición verdaderos, con una $R^{2}$ mediana de 0,2 (9). La variación de la sensibilidad y especificidad ha sido igualmente amplia (9). Los mejores datos se obtienen cuando los agentes a los que hay exposición se perciben con facilidad; cuando se usan términos familiares habitualmente empleados en los lugares de trabajo, o los nombres comerciales, productos o grupos de sustancias y productos que el encuestado ha comprado o seleccionado personalmente (9). Mostrarle al entrevistado una lista de productos, sustancias o grupos de sustancias puede servir para mejorar su percepción e identificación de los agentes. Este sería el caso, por ejemplo, de los plaguicidas usados por los agricultores $(7,9,10)$.

$\mathrm{Si}$ bien las posibilidades de obtener datos sobre las exposiciones a agentes específicos son limitadas, las entrevistas a los participantes en estudios de casos y testigos constituyen la ocasión más idónea para recoger datos sobre los factores condicionantes de las exposiciones. El porcentaje de correspondencia entre los autoinformes y los registros de las empresas sobre estos factores condicionantes básicos, tales como el tipo de trabajo, la antigüedad en el puesto de trabajo y las fechas de comienzo y finalización, oscila entre 70 y $90 \%$ o más (9). La validez de los datos obtenidos por medio de entrevistas es menor en el caso de historiales ocupacionales complejos o los correspondientes a puestos de muy corta duración (meses) o a los puestos más antiguos dentro de una larga historia laboral (9). En las entrevistas se pueden recolectar datos acerca de una gran variedad de condicionantes espe- cíficos (cuadro 1), pero la validación de estos datos retrospectivos suele ser difícil de realizar.

El empleo de algunos métodos para determinar la exposición, como el uso de iconos para ilustrar las formas de aplicar la sustancia nociva, la duración de las tareas y de los puestos, las circunstancias en que se produjo la exposición, el equipo de protección personal utilizado y otros elementos condicionantes de la exposición, han demostrado tener claras ventajas en las entrevistas de trabajadores agrícolas en Costa Rica y de inmigrantes hispanoamericanos en los Estados Unidos (11-13).

En dos estudios en los que se utilizaron encuestas verbales, el sesgo de recordación (recall bias) fue similar entre casos y testigos. La sensibilidad a los parámetros de evocación de las exposiciones y de sus factores condicionantes fue idéntica $(9,14)$.

La experiencia de expertos en higiene industrial o en ambiente laboral es un elemento necesario para la formulación de los cuestionarios. Las entrevistas deben estar sujetas a un control de calidad (4).

Bioindicadores de exposición. Por otro lado, algunos bioindicadores de exposición, tales como las concentraciones de sustancias o de sus metabolitos o aductos de ADN en medios biológicos humanos, pueden considerarse marcadores de la exposición pasada. Dependiendo del tiempo que dure el agente o su metabolito en el organismo, podría ser necesario recurrir a mediciones pasadas a fin de poder establecer la relación entre el agente y la exposición a él durante el período de evaluación. La factibilidad de recolectar biomarcadores de exposición depende del número de sujetos estudiados y de consideraciones bioéticas y socioéticas, al igual que del desarrollo de técnicas analíticas estandarizadas para el análisis del bioindicador. Dado que los bioindicadores de sustancias que desaparecen del organismo en poco tiempo por lo común reflejan exposiciones relativamente recientes, podrían no ser aptos para evaluar la exposición retrospectivamente. Por otro lado, si los agen- tes perduran en el organismo largo tiempo, es posible que reflejen las exposiciones de todas las fuentes durante un período prolongado, pudiendo interferir con las interpretaciones de las exposiciones ocupacionales. Por ejemplo, los bioindicadores de hidrocarburos poliaromáticos reflejan las exposiciones a fuentes ocupacionales, al humo de tabaco y a la contaminación del aire ambiental durante distintos períodos y causan confusión al interpretar las fuentes, así como los períodos de latencia (15). Además, los bioindicadores que perduran en el organismo tienden a reflejar exposiciones que ocurren en diferentes períodos, lo cual dificulta la determinación de la exposición durante el período de interés etiológico para el estudio. Como la variación intraindividual de bioindicadores suele ser grande, se aumentan los costos y se reduce la población objeto de estudio.

No obstante, se han aplicado, con buenos resultados, bioindicadores de exposición en estudios etiológicos sobre el cáncer, siendo un ejemplo el de estudios de exposición al diclorodifeniltricloroetano (DDT) en pacientes con cáncer de mama (16). El DDT y sus metabolitos se acumulan y perduran en los tejidos grasos y pueden medirse en la sangre. Por consiguiente, la concentración de estos compuestos en los casos y en los testigos refleja sus exposiciones pasadas, incluso después de una década o más desde la prohibición de este insecticida. Por supuesto, este bioindicador refleja las exposiciones de todo tipo: ocupacionales, ambientales y alimentarias. Entre 1995 y 1996, Olaya-Contreras et al. (17) dirigieron un estudio de casos y testigos en Santa Fe de Bogotá, Colombia, que abarcó a 153 pacientes de cáncer de mama y a 153 testigos pareados con los casos por edad. En este estudio no fue necesario usar ningún dato secundario. En el cuadro 2 se presentan las razones de posibilidades (odds ratios, RP) según la concentración del metabolito diclorodifenildicloroetano (DDE), con un riesgo significativamente alto en la categoría de mayor exposición.

Datos secundarios. Los datos secundarios de las exposiciones o de sus fac- 
CUADRO 2. La razón de posibilidades (RP) de sufrir cáncer de mama, en función de la concentración de diclorodifenildicloroetileno (DDE) en la sangre, con ajustes según la lactancia del primer niño, edad materna en el primer parto, antecedentes familiares de cáncer de mama, índice de masa corporal y presencia de la menopausia. Santa Fe de Bogotá, Colombia, 1995-1996

\begin{tabular}{ccll}
\hline $\mathrm{DDE}(\mathrm{ng} / \mathrm{mL}$ de suero) & No. de casos & $\mathrm{RP}$ & IC95\% $^{\mathrm{a}}$ \\
\hline $0,10-0,14$ & 39 & 1,0 & \\
$0,15-1,96$ & 45 & 1,20 & $0,64-2,25$ \\
$1,97-19,2$ & 69 & 1,95 & $1,10-3,52$ \\
\hline
\end{tabular}

Fuente: Referencia 17

a IC95\%: Intervalo de confianza del 95\%.

tores condicionantes provienen de fuentes externas a la población objeto de estudio y son de tipo agregado o agrupado, no individual. La recolección de datos secundarios es cada vez más común y no se lleva a cabo mediante entrevistas, sino que se efectúa directa o indirectamente a partir de las circunstancias particulares de grupos o poblaciones de trabajadores. Las fuentes de datos secundarios que se utilizan son, entre otras, las siguientes: fuentes bibliográficas, estudios de campo, documentos de industrias o empresas (sobre tecnologías, procesos, materiales, productos, protección), elementos de protección personal y otras medidas de control, informantes de industrias o empresas, supervisores, extensionistas, trabajadores experimentados, trabajadores jubilados, representantes de trabajadores, vendedores, mediciones de exposición, otros sistemas de vigilancia, informes de administración laboral (inspección), estadísticas laborales, industriales y de importaciones y documentos e informantes del ámbito sindical. Constituyen un ejemplo, los datos de un estudio de casos y testigos de leucemia infantil en Costa Rica, los cuales se recolectaron de dos formas. Los datos primarios y los relativos a los factores condicionantes de las exposiciones a plaguicidas se obtuvieron por entrevistas a padres y madres mediante métodos tradicionales y un sistema de iconos visuales. Dado que la calidad de los datos sobre exposiciones anteriores a plaguicidas específicos no era suficiente para elaborar indicadores de exposición individual a estos compuestos entre 1980 y 2000, a modo de datos ex- ternos secundarios se utilizaron datos sobre el uso de diferentes plaguicidas en cultivos, períodos y regiones de Costa Rica procedentes de bases de datos del Ministerio de Agricultura, registros bancarios y otras oficinas especializadas (18). Los datos primarios y secundarios de este estudio se integraron mediante un modelo de exposición para determinar cuáles habían sido los niveles de exposición de los padres y las madres a 27 plaguicidas en diversas ventanas de tiempo (véase la sección "Modelos de exposición").

Se deduce que los tipos y las fuentes de datos secundarios son más diversos, pudiendo abarcar desde los conocimientos generales documentados sobre las características químicas y físicas de los agentes, su toxicidad, potencial cancerígeno, mutagenicidad, teratogenicidad, bioacumulación y vías de ingreso, hasta otras fuentes y tipos más específicos, como las descripciones del proceso de producción usado, la probabilidad y los niveles de exposición en distintos ambientes ocupacionales, las cantidades de materiales y productos usados o producidos en ellos, los controles de ingeniería observados, las características de la maquinaria y el equipo y las condiciones de ventilación y de temperatura. De vez en cuando se dispone de datos, en el ámbito de la higiene industrial, para entornos específicos o para ambientes con condiciones similares, de los cuales será preciso extrapolar los resultados de las mediciones ambientales en el espacio y en el tiempo. Por ejemplo, en un estudio de cohorte realizado en ocho países europeos sobre el riesgo de cáncer en trabajadores de fábricas de asfalto, se recolectaron todos los datos correspondientes a mediciones de higiene industrial que estaban disponibles (número de mediciones $=2$ 007) sobre los compuestos usados en el pavimento de carreteras en estos países durante diferentes períodos (19; véase la sección "Modelos de exposición").

Como ya se ha señalado, la mayor limitación de los datos secundarios radica en su carácter agregado; utilizar un solo valor de exposición para todos los miembros de un grupo de personas expuestas a un factor de riesgo lleva a estimaciones falsas de las exposiciones individuales. Los modelos estadísticos jerárquicos ofrecen posibilidades avanzadas para el análisis de los datos de este tipo.

\section{Determinación del historial de exposición}

Se pueden distinguir tres grandes grupos de métodos para determinar el historial de exposición: las matrices de exposición, la evaluación a cargo de expertos, y los modelos de exposición, con algún grado de superposición entre los tres métodos.

Matrices de exposición. Una matriz de exposición es un arreglo o configuración multidimensional definida por uno o más factores condicionantes de la exposición y por una serie de agentes (químicos, físicos o biológicos). Se pueden insertar uno o más indicadores de la exposición en cada celda. Las primeras matrices consistieron en clasificaciones cruzadas de un factor condicionante (sector económico $u$ ocupación) y de una serie de agentes nocivos, entre ellos algunas sustancias cancerígenas $(3,20,21)$. Estas matrices se aplicaron para calcular la probabilidad de exposición a partir de los sectores u ocupaciones. Estos sistemas se llamaron "matrices de exposición laboral" (en inglés, job exposure matrices, JEM), debido a que en ellos se utilizaban predominantemente sectores económicos y ocupaciones.

Las matrices de exposición modernas permiten incluir múltiples factores condicionantes: sector económico, ocu- 
pación, tarea o puesto, período, empresa, zona geográfica y sexo, entre otros. En una matriz, la población de referencia puede estar integrada por los trabajadores de una empresa o de una región, o bien, incluir la fuerza de trabajo nacional entera como referencia directa. Los indicadores que figuran en las celdas (combinaciones de factores condicionantes) de la matriz pueden ser las proporciones de personas expuestas (probabilidad de exposición); el nivel promedio de la exposición o la categoría de la exposición según su intensidad; la fracción promedio o la clasificación del tiempo de exposición (clasificada en términos de la fracción de la jornada laboral implicada [p. ej., menos del 5\%, del 5 al 30\%, más del $30 \%$, etc.]); el promedio de la exposición acumulada o categorizada; la presencia e intensidad de las exposiciones pico; así como uno o más indicadores de la fiabilidad de la información que figura en cada celda [grado de certeza de la exposición: confiable, probable o incierto, pero posible].

Recientemente, para estimar el número de trabajadores expuestos a agentes cancerígenos y plaguicidas en Costa Rica (22) se construyó una matriz denominada TICAREX que era una adaptación del sistema de datos europeo sobre la exposición a agentes cancerígenos CAREX (CARcinogenic EXposure) $(23,24)$. Esta matriz, basada en una combinación de mediciones efectuadas en los Estados Unidos y Finlandia y en otros datos aporta-dos por expertos, la están modificando expertos nacionales en Costa Rica. TICAREX incluye dos factores condicionantes: el sector económico (55 clases) y el sexo. Los agentes son 27 sustancias cancerígenas importantes en Costa Rica y 7 grupos de plaguicidas. En CAREX y TICAREX, los elementos colocados en las celdas son las proporciones de personas expuestas. TICAREX es una matriz nacional que se aplica a todos los trabajadores mayores de 12 años de edad, incluidos los del sector informal, y se refiere al año 2000. En Costa Rica, las exposiciones más frecuentes a agentes cancerígenos resultaron ser la radiación solar, las emisiones de diesel, el humo ambiental de tabaco, el benceno y los compuestos hexavalentes de cromo; las exposiciones más frecuentes a plaguicidas resultaron ser al paraquat, diquat, mancozeb, maneb y zineb. El recorrido de la prevalencia de estas exposiciones frecuentes fue de $0,4 \%$ a $25 \%$ (22).

Un grupo de expertos finlandeses con experiencia en la evaluación de exposiciones a agentes físicos, químicos, microbiológicos, ergonómicos y psicosociales construyó una matriz nacional, denominada Finlandia Job Exposure Matrix (FINJEM) (25), que da las proporciones de personas expuestas y los niveles promedio de exposición a 74 agentes, con arreglo a dos factores condicionantes: la ocupación (311 clases) y el período calendario (cuatro actualizaciones realizadas desde su creación en 1983). En la elaboración de esta matriz se usó una base de datos con mediciones de higiene industrial para agentes químicos, físicos, biológicos y ergonómicos, junto con la evaluación por expertos. La FINJEM se actualiza cada tres años y se ha aplicado en varios estudios epidemiológicos, entre ellos uno sobre el cáncer de páncreas y dos sobre las exposiciones laborales a agentes potencialmente cancerígenos en Finlandia (26) y España (27).

Las matrices TICAREX y FINJEM son nacionales. Existen también matrices más restringidas, construidas para un estudio específico. El Instituto Nacional del Cáncer de los Estados Unidos elaboró una matriz de exposición a hidrocarburos alifáticos clorados para un estudio de casos y testigos sobre el cáncer del cerebro en tres estados de ese país (28). Los agentes seleccionados fueron: tetracloruro de carbono (tetraclorometano), cloroformo (triclorometano), cloruro de metileno (diclorometano), 1,1,1-tricloroetano (cloroformo metilo), tricloroetileno y tetracloroetileno. Se incluyeron tres factores condicionantes: tipo de industria, ocupación y década. Los valores que figuran en las celdas son los de las probabilidades semicuantitativas (baja/media/alta) de exposición y de las tres intensidades de exposición. Algunas celdas permanecen vacías para las combinaciones industria-ocupación-década dado que no existían casos o testigos con esa información.
Las principales fuentes para la elaboración y la actualización de las matrices de exposición laboral son: la evaluación a cargo de expertos, las fuentes bibliográficas, los archivos industriales, personas expertas (gerentes, encargados de ventas, trabajadores de mayor antigüedad o experiencia, supervisores, representantes de los trabajadores y jefes de producción, entre otros, las observaciones directas, las mediciones de exposición y las bases de datos de exposiciones). Por ejemplo, los datos de la matriz TICAREX tienen su origen en mediciones propias de la higiene industrial, estudios de campo en lugares de trabajo y evaluaciones por expertos de Costa Rica, Finlandia y Estados Unidos.

La limitación más grave de las matrices de exposición es su carácter agregado. El sujeto de cada celda comparte el valor del indicador de exposición de dicha celda, ya sea este la probabilidad o intensidad de la exposición o cualquier otro. Como resultado, se pierde la variabilidad de la exposición dentro de las celdas y se producen diferentes grados de clasificación errónea de las exposiciones individuales, así como sesgos en las estimaciones del riesgo. La sensibilidad de las matrices es baja $(<0,5)$, o sea que se producen muchos resultados negativos falsos. La especificidad es habitualmente más alta $(>0,8)$ y los resultados positivos falsos son relativamente pocos $(9,21)$. El efecto de las clasificaciones erróneas sobre los indicadores de riesgo puede ilustrarse con el siguiente ejemplo. Supongamos que la prevalencia de la exposición sea de $10 \%$, la sensibilidad, de $70 \%$, la especificidad, de $80 \%$ y la razón de posibilidades (RP) corregida, de 4,0. Con estos valores de sensibilidad, especificidad y prevalencia de la exposición, se puede calcular una RP esperada de 1,8. Este sesgo es resultado principalmente de la baja especificidad (80\%). Cuando las prevalencias de las exposiciones son bajas $(<0,1)$, que es la situación habitual en la población, poder lograr un máximo de especificidad en los indicadores de exposición suele ser un factor decisivo. Algunos métodos para determinar el sesgo los describen Klein- 
baum, Kupper y Morgenstern (29), y hay otras presentaciones gráficas en publicaciones de T. Partanen (30).

La validez de los datos de la matriz usada para determinar la relación entre la exposición y los riesgos para la salud dependerá de la validez de las fuentes de los datos usados para construir la matriz. En general, se subestiman las asociaciones con matrices de poca validez.

Obviamente, una matriz que permite usar varios factores condicionantes específicos funciona mejor que una matriz más general. Dosemeci et al. (28) demostraron un aumento del indicador del riesgo (razón de posibilidades, RP) de astrocitoma en conexión con la exposición al cloruro de metileno al introducir factores condicionantes adicionales en el análisis. Así, la RP aumentó de 1,5 (sin tener en cuenta la probabilidad de exposición) a 2,5 (cuando se contempló una alta probabilidad de exposición); pero al incluir en el modelo las clasificaciones de los sectores económicos y ocupaciones, la RP aumentó hasta 4,2, y al hacerse el ajuste adicional según la década, la RP aumentó a 6,1.

Merece la pena mencionar las enormes bases de datos empíricas de exposiciones y factores condicionantes recopiladas por el Instituto Nacional de Seguridad y Salud Ocupacional (National Institute of Occupational Safety and Health, NIOSH) de los Estados Unidos (31-34). Llegado el caso, dichas bases de datos podrían transformarse en matrices de exposición.

La evaluación a cargo de expertos. Los expertos en exposiciones realizan este tipo de determinación con arreglo a principios predeterminados. Combinan los datos primarios procedentes de historiales laborales individuales, usualmente obtenidos mediante entrevistas, así como los datos secundarios procedentes de algunas de las fuentes mencionadas anteriormente, teniendo en cuenta la calidad del dato, la disponibilidad de la información y los recursos existentes. Uno o más expertos establecen los principios y las condiciones necesarias y proceden a la evaluación a fin de convertir los datos primarios y secundarios en indicadores de exposición. La evaluación a cargo de expertos es menos mecánica que las matrices de exposición, porque el experto puede modificar el algoritmo durante el proceso de evaluación y buscar datos adicionales primarios o secundarios cuando sea conveniente y viable. Los expertos pueden ser higienistas industriales, químicos, ingenieros, técnicos y otros profesionales con conocimientos específicos en el campo de la evaluación; estos profesionales utilizan una gran variedad de fuentes de datos: consideraciones teóricas, datos probatorios anteriores y entrevistas ad hoc de informantes clave, entre otros (35).

Este método de evaluación a cargo de expertos se empleó en el estudio de casos y testigos sobre el cáncer ocupacional más grande que jamás se haya realizado en el mundo $(7,8)$. Es importante resaltar la metodología utilizada para determinar y caracterizar la exposición ocupacional a agentes cancerígenos ocupacionales. Se estudiaron de forma prospectiva todos los hombres entre los 35 y 70 años de edad que residían en Montreal, Canadá, y que estaban hospitalizados con un diagnóstico de cáncer en cualquiera de 19 localizaciones anatómicas consideradas; se excluyeron las leucemias y algunos cánceres poco frecuentes. Se seleccionaron testigos de la población en general y entre pacientes con otros tipos de cáncer. Se entrevistó a cada persona mediante un procedimiento detallado semiestructurado para recoger sus historiales ocupacionales completos, específicamente los materiales y procesos de producción, lugares de trabajo, maquinaria, organización del trabajo, medidas de protección y otros factores condicionantes de su exposición. La entrevista fue sumamente elaborada y constó de un cuestionario que debía completarse previamente, módulos para trabajos específicos (jobspecific modules) y listas de control. Un grupo de 10 higienistas industriales y químicos examinaron y codificaron cada entrevista según los principios previamente elaborados. Se codificaron 275 agentes (sólidos, gases, humos, líquidos, vapores), familias de sustancias químicas, categorías generales (solventes, plaguicidas, aminas, metales, radiación, etc.), forma de contacto (respiratoria, cutánea, o ambas), nivel de exposición (bajo/promedio/ alto), frecuencia de la exposición $(<5 \%$, del 5 al $30 \%$ y del 30 al $100 \%$ de la jornada laboral de 8 horas) y grado de confianza referente a la exposición (posible/probable/absoluto). Para realizar esta codificación los expertos usaron como fuentes de datos secundarios de publicaciones científicas y técnicas, de publicaciones industriales, entrevistas anteriores a personas en ocupaciones similares, consultas con expertos externos y asociaciones industriales. El número promedio de agentes por ocupación fue de 8,1, y las exposiciones más frecuentes tuvieron lugar en relación con hidrocarburos policíclicos, monóxido de carbono, compuestos a base de plomo, emisiones de motores y solventes. Se llevó a cabo un extenso análisis de riesgos, y la mayoría de los resultados se publicaron en una monografía (8).

Como puede deducirse, la calidad de la determinación del historial de exposición depende del grado de experiencia o calidad de los expertos, de los datos primarios y secundarios, de los compuestos específicos, del número de detalles y del proceso de evaluación. En las evaluaciones sobre la coherencia entre la evaluación a cargo de expertos y las mediciones de higiene industrial se ha informado de coeficientes de correlación $r^{2}$ que oscilan entre 0 y 0,62 , en función del agente y de los detalles de los procedimientos. La sensibilidad del método de evaluación a cargo de expertos ha variado entre 0,21 y 0,79 , pero su especificidad es elevada $(0,91-$ $0,98)$. La validez de la evaluación de los expertos mejora cuando se dispone de datos secundarios correspondientes a mediciones de parámetros de higiene industrial (9). La regularidad de la evaluación puede determinarse comparando las evaluaciones de varios expertos. La concordancia entre expertos independientes es de aproximadamente $90 \%$ en relación con la presencia (sí-no) del compuesto (36), pero puede ser mucho más baja en el caso de indicadores semicuantitativos o cuantitati- 
CUADRO 3. Pesos generales $\left(w_{\mathrm{i}}\right)$ y valores de peligro $\left(H_{\mathrm{i}}\right)$ de los factores condicionantes (i) de la exposición

\begin{tabular}{lcl}
\hline Factor condicionante (i) & $W_{\mathrm{i}}$ & $H_{\mathrm{i}}$ \\
\hline Puesto y tecnología & 0,40 & $0-5^{\mathrm{a}}$ \\
Protección personal & 0,20 & $0-5$ \\
Reingreso al área ya tratada & 0,10 & $0-5$ \\
Almacenaje de plaguicidas & 0,15 & $0-5$ \\
Higiene personal & 0,15 & $0-5$ \\
$\quad$ Total & 1,00 & \\
\hline
\end{tabular}

Fuente: Referencia 37.

${ }^{a} H_{i}=0$ : ninguna exposición; $H_{i}=5$ : peligro muy alto.

CUADRO 4. Valores de peligro $\left(H_{\mathrm{i}}\right)$ para distintas categorías del factor condicionante "puesto y tecnología" en la agricultura

\begin{tabular}{|c|c|}
\hline Categoría de puesto & $H_{\mathrm{i}}$ \\
\hline Aplicación manual & 4 \\
\hline $\begin{array}{l}\text { Trabajador encargado de la fumigación } \\
\text { en el aeropuerto }\end{array}$ & 4 \\
\hline Mezclador profesional & 4 \\
\hline $\begin{array}{l}\text { Tareas con aplicación de plaguicidas } \\
\text { o contacto directo evidente } \\
\text { (11 subcategorías, por ejemplo, } \\
\text { aplicador no manual) }\end{array}$ & 3 \\
\hline $\begin{array}{l}\text { Tareas en campo aplicado sin uso directo } \\
\text { de plaguicidas (16 subcategorías, por } \\
\text { ejemplo empacadora) }\end{array}$ & 2 \\
\hline $\begin{array}{l}\text { Trabajo con posible exposición a plagui- } \\
\text { cidas (18 subcategorías, por ejemplo, } \\
\text { tractorista) }\end{array}$ & 1 \\
\hline $\begin{array}{l}\text { Trabajo sin exposición a plaguicidas } \\
\text { (3 subcategorías, por ejemplo, } \\
\text { agricultura orgánica) }\end{array}$ & 0 \\
\hline
\end{tabular}

Fuente: Referencia 37.

vos. Se puede mejorar la coherencia interna si se elaboran criterios formales estrictos para la evaluación (3).

Evidentemente, la evaluación a cargo de expertos es un método recomendable, pero puede incrementar considerablemente los costos en función de la magnitud del estudio.

Modelos de exposición. Un modelo de exposición permite predecir o estimar el indicador de exposición a partir de los valores de los factores condicionantes de la exposición. Los datos correspondientes a los factores condicionantes pueden ser primarios, secundarios o ambos. Los modelos adoptan una forma de función determinística o estocástica que es computadorizada.
Burstyn et al. (19) elaboraron un modelo de exposición en obreros del asfalto en Alemania, Dinamarca, Finlandia, Francia, Holanda, Israel y Suecia. Se trata de un estudio de cohorte con 79822 obreros de estos países a los que se les hizo un seguimiento para determinar su mortalidad por cáncer y otras causas durante el período de 19532000. Se llevó a cabo una evaluación cuantitativa de exposición a humo negro, vapores orgánicos e hidrocarburos policíclicos aromáticos, así como una evaluación semicuantitativa de la exposición a emisiones de diesel, amianto (asbesto), polvo de silicio y alquitrán de carbón.

En la evaluación cuantitativa se utilizó una fórmula empírica de la regresión lineal, a saber, una base de datos de 2007 mediciones de exposición como variable dependiente, y varios valores de los factores condicionantes, tales como pavimento con almáciga (resina derivada del árbol Pistacia lenticus) bituminosa; uso de grandes cantidades de ligante; interacción entre la almáciga y el desempeño de un trabajo o una tarea con la máxima exposición; reciclaje del asfalto; interacción entre el reciclaje y un trabajo o una tarea con la máxima exposición; tratamiento del pavimento con asfalto líquido; grava con aceite o alquitrán, año calendario y temperatura de la aplicación. Se calculó la exposición promedio para los diferentes escenarios durante intervalos de tiempo como la suma de productos de los niveles de exposición durante cada escenario y de las fracciones de tiempo de exposición para cada escenario. Para calcular la exposición promedio para los diferentes escenarios e intervalos de tiempo, se multiplicó el valor del nivel de exposición por la fracción de tiempo correspondiente a cada escenario y finalmente se sumaron estos productos para obtener un total acumulado de la exposición.

Otro ejemplo fue la elaboración, en un estudio de casos y testigos sobre la leucemia infantil en Costa Rica, de un modelo de la exposición ocupacional de padres y madres a los plaguicidas (37). Se recolectaron los datos primarios sobre las exposiciones y factores condicionantes en las entrevistas, usando tanto un cuestionario convencional como el formato pictórico mencionado anteriormente. Los años informados se dividieron en cinco períodos: el año antes de la concepción, los tres trimestres del embarazo y el primer año de vida del niño.

Los datos de las entrevistas sobre cultivos, plaguicidas, períodos de uso y factores condicionantes de la exposición se adaptaron en estos cinco períodos. Los datos secundarios se obtuvieron de datos externos (18) sobre las dosis agrícolas utilizadas, expresadas en litros de ingrediente activo por hectárea (L/ha). Para el modelo se seleccionaron 27 plaguicidas, 14 cultivos, un período total de 21 años y 14 regiones geográficas. Se utilizaron los datos primarios de cinco factores condicionantes (puesto y tecnología usada, protección personal, regreso al área tratada previamente con plaguicidas, forma de almacenamiento de los plaguicidas e higiene personal), cada uno representado por categorías múltiples (cuadros 3 y 4).

Los valores correspondientes al peligro relacionado con cada categoría de cada factor condicionante y el peso general otorgado a cada factor condicionante se decidieron a priori por consenso entre los investigadores.

El índice individual de factores condicionantes durante un período es la suma de productos de los valores de peligro y sus respectivos pesos. Se multiplica el índice individual por la dosis agrícola (L/ha) pertinente, la cual sirve de indicador promedio individual de la intensidad de la exposición, que oscila entre ninguna exposición y una exposición elevada. Este producto es el indicador promedio individual de la intensidad de la exposición a plaguicidas en un período determinado.

Contrariamente a los estudios transversales de validación de los factores condicionantes de exposiciones a plaguicidas, Arbuckle et al. (38), en el estudio costarricense, no han individualizado datos para la validación directa del modelo. Además, considerando que la vía de ingreso por piel es decisiva para definir la exposición a plaguicidas, no se separaron las exposiciones respiratorias de las cutáneas (3). Mediante la utilización de ocho factores condicionantes, 
Brouwer et al. (39) formularon un modelo inusual de exposiciones prolongadas a plaguicidas que contempla explícitamente las exposiciones cutáneas.

Como los modelos de exposiciones son formales y rígidos, permiten un cómputo relativamente completo (34, 37 ), lo cual hace que el costo de su aplicación sea relativamente barato. Los problemas de validación permanecen, ya que usualmente es difícil establecer muestras de poblaciones para las cuales se tengan valores de factores condicionantes y exposiciones verdaderas y suficiente variación en las exposiciones y factores condicionantes.

Teóricamente, los modelos ofrecen las mejores posibilidades de evaluar las exposiciones personales. Obviamente, pueden presentarse dificultades con la validación directa, la disponibilidad y validez de los datos primarios y secundarios, y la forma del modelo. El análisis de sensibilidad ofrece la posibilidad de realizar una validación indirecta. Se puede cambiar la forma, o uno o más de los parámetros del modelo, y determinar la influencia de los cambios en el índice de exposición. La selección del modelo final (forma y parámetros) puede ser problemática. Se pueden introducir consideraciones fisiológicas y mecanicistas para la selección final.

\section{CONCLUSIÓN}

La determinación del historial de exposiciones personales tiene gran utili- dad en la epidemiología ocupacional, pues sirve para caracterizar la exposición, comparar la exposición de las distintas personas expuestas, estimar la sobreexposición, así como estimar la asociación entre la exposición y ciertos riesgos para la salud. Los principales problemas guardan relación con las fuentes de datos, la calidad de los mismos, la cuantificación de los niveles de exposición, los parámetros temporales de la exposición, y la factibilidad y el uso de los datos secudarios y agregados. Actualmente se está avanzando aceleradamente en el conocimiento de estos puntos, y la determinación de la exposición actual y pasada es una práctica que se ha extendido en el mundo entero con gran rapidez.

Hemos abordado las fuentes de datos primarios y secundarios acerca de las exposiciones y sus factores condicionantes, así como otros métodos diferentes para determinar las exposiciones. La validez y coherencia de los datos obtenidos de distintas fuentes y de los métodos aplicados para determinar la exposición varían enormemente (9), incluso dentro de un único conjunto de datos (40), y en el estimador final de la exposición personal se acumulan las deficiencias de sus componentes. Por ende, la interpretación de los indicadores de exposición resultantes depende directamente de la validez de sus diferentes componentes.

Dado que no existen mediciones de las exposiciones ambientales y biológicas anteriores, los autoinformes siguen

\section{REFERENCIAS}

1. Kauppinen TP. Assessment of exposure in occupational epidemiology. Scand J Work Environ Health. 1994;20:19-29.

2. Stewart P. Challenges to retrospective exposure assessment. Scand J Work Environ Health.1999;25:505-10.

3. Vermeulen R, Stewart P, Kromhout H. Exposure assessment in occupational epidemiologic research. Scand J Work Environ Health. 2002;28:371-85.

4. Stewart WF, Stewart PA. Occupational casecontrol studies I: Collecting information on work histories and work-related exposure. Am J Ind Med. 1994;26:297-312.

5. Stewart PA, Stewart WF. Occupational casecontrol studies II: recommendations for exposure assessment. Am J Ind Med. 1994;26: 313-26.
6. Stewart PA, Stewart WF, Siemiatycki J, Heineman EF, Dosemeci M. Questionnaires for collecting detailed occupational information for community-based case-control studies. Am Ind Hyg Ass J. 1998;58:39-44.

7. Gérin M, Siemiatycki J, Kemper H, Bégin D. Obtaining occupational exposure histories in epidemiologic case-control studies. J Occup Med. 1985;27:420-6.

8. Siemiatycki J. Risk factors for cancer in the workplace. Boca Raton, FL: CRC Press; 1991.

9. Teschke K, Olshan AF, Daniels JL, De Roos AJ, Parks CG, Schulz M, et al. Occupational exposure assessment in case-control studies: opportunities for improvement. Occup Environ Med. 2002;59:575-94.

10. Blair A, Stewart P, Kross B, Ogilvie L, Burmeister L, Ward M, et al. Comparison of siendo decisivos para obtener datos primarios, y los datos secundarios se utilizarán con mayor frecuencia que antes. Las matrices de exposición laboral, para la población en general o la dedicada a una actividad específica, están cobrando cada vez mayor importancia en la epidemiología ocupacional. Esto se debe a que permiten detectar con más facilidad cualquier relación causal entre la exposición y la enfermedad - gracias a un mejor registro de los factores que determinan la exposición-, y darles prioridad a medidas de vigilancia epidemiológica teniendo en cuenta la proporción de la fuerza laboral expuesta a factores cancerígenos. La evaluación a cargo de expertos es probablemente el método que puede alcanzar la mayor validez, pero puede acarrear costos muy altos en estudios medianos o grandes. Una buena opción es la de utilizar un modelo formal que evalúe las exposiciones obvias y la evaluación a cargo de expertos en situaciones en que los resultados del modelo no parecen ser obvios o realistas. En general, puede considerarse la posibilidad de utilizar, en mayor o menor grado, combinaciones de diferentes fuentes de datos y distintos métodos para determinar las exposiciones.

Agradecimiento. Los autores tienen una deuda de agradecimiento con las agencias Colciencia y Terry Fox, que aportaron fondos para un proyecto del cual formó parte este estudio. two techniques to obtain information on pesticide use from Iowa farmers by interview. J Agricult Safety Health. 1997;3:229-36.

11. Monge $\mathrm{P}$, Wesseling $\mathrm{C}$, Engel L, Rojas $\mathrm{M}, \mathrm{Zu}-$ urbier M, Keifer M, et al. An icon based interview for the assessment of parental occupational pesticide exposure in a case-control study of childhood leukemia. Int J Occup Health. 2004;10:84-90.

12. Engel L S, Keifer MC, Zahm SH. A comparison of a traditional questionnaire with an icon/calendar based questionnaire to assess occupational history. Am J Ind Med. 2001;40: 502-11.

13. Zahm SH, Colt J, Engel LS, Keifer MC, Alvarado AJ, Burau K, et al. Development of a life events/icon calendar questionnaire to ascertain occupational histories and other char- 
acteristics of migrant farmworkers. Am J Ind Med. 2001;40:490-501.

14. Rothman KJ, Greenland S. Modern Epidemiology. Second edition. Philadelphia: LippincottRaven Publishers; 1998.

15. Pearce N, de Sanjosé S, Boffetta P, Kogevinas M, Saracci R, Savitz D. Limitations of biomarkers of exposure in cancer epidemiology. Epidemiology. 1995;6:190-4.

16. Welp EA, Weiderpass E, Boffetta $P$, Vainio $H$, Vasama-Neuvonen K, Petralia S, et al. Environmental risk factors of breast cancer. Scand J Work Environ Health. 1998;24:3-7.

17. Olaya-Contreras P, Rodríguez-Villamil J, Posso-Valencia HJ, Cortez JE. Organochlorine exposure and breast cancer in Colombian women. Cad Saude Publica (Rio de Janeiro). 1998;14:125-32.

18. Wesseling C, Bravo V. Pesticide use on the main crops of Costa Rica 1970-2000. Data for retrospective exposure assessment in epidemiologic studies. Report to the US National Cancer Institute. Heredia, Costa Rica: Central American Institute for Studies on Toxic Substances (IRET), Universidad Nacional; 2002.

19. Burstyn I, Boffetta P, Kauppinen T, Heikkila P, Svane O, Partanen T, et al. Estimating exposures in the asphalt industry for an international epidemiological cohort study of cancer risk. Am J Ind Med. 2003;43:3-17.

20. Hoar SK, Morrison AS, Cole P, Silverman DT. An occupational linkage system for the study of occupational carcinogens. J Occup Med. 1980;22:722-6.

21. Kromhout H, Vermeulen R. Temporal, personal and spatial variability in dermal exposure. Ann Occup Hyg. 2001;45(4):257-73.

22. Partanen $T$, Chaves J, Wesseling $C$, Chaverri F, Monge P, Ruepert C, et al. Workplace carcinogen and pesticide exposures in Costa Rica. Int J Occup Environ Health. 2003;9: 104-11.

23. Kauppinen T, Toikkanen J, Pedersen D, Young R, Ahrens W, Boffetta P, et al. Occupational exposure to carcinogens in the Euro- pean Union. Occup Environ Med. 2000;57: $10-8$.

24. Kogevinas M, Maqueda J, de la Orden V, Fernández F, Kauppinen T, Benavides FG. Exposición a carcinógenos laborales en España: aplicación de la base de datos CAREX. Arch Prev Riesgos Labor. 2000;3:153-9.

25. Kauppinen T. Finnish occupational exposure databases. Appl Occup Environ Hyg. 2001;16: $154-8$.

26. Kauppinen T, Partanen T, Degerth R, Ojajärvi A. Pancreatic cancer and occupational exposures. Epidemiology. 1995;6:498-502.

27. Alguacil J, Kauppinen T, Porta M, Partanen T, Malats N, Kogevinas M, et al. Risk of pancreatic cancer and occupational exposures in Spain. PANKRAS Study Group. Ann Occup Hyg. 2000;5:391-403.

28. Dosemeci M, Cocco P, Gomez M, Stewart PA, Heineman EF. Effects of three features of a job-exposure matrix on risk estimates. Epidemiology. 1994;5:124-7.

29. Kleinbaum DG, Kupper LL, Morgenstern H. Epidemiological research. Principles and quantitative methods. Belmont: Lifetime Learning; 1982.

30. Partanen T. Effect of misclassification on odds ratio. In: Gudbergsson H, Jarvholm B, Kauppinen T, Kjuus H, Lynge E, Olsen J, et al. Use of job exposure matrices in the Nordic countries. Copenhagen: Kraeftens Bekaempelse, Cancerregistere; 1988.

31. Sieber WK, Sundin DS, Frazier TM, Robinson CF. Development, use, and availability of a job exposure matrix based on national occupational hazard survey data. Am J Ind Med. 1991;20:163-74.

32. Greife A, Young R, Carroll M, Sieber WK, Pedersen D, Sundin D, et al. National Institute for Occupational Safety and Health general industry occupational exposure databases: their structure, capabilities, and limitations. Appl Occup Environ Hyg. 1995;10:264-9.

33. Boiano JM, Hull RD. Development of a national occupational exposure survey and database associated with NIOSH hazard surveillance initiatives. Appl Occup Environ Hyg. 2001;16:128-34.

34. Hughson GW, Cherrie JW. Validation of the EASE expert system for dermal exposure to zinc. In: Hagberg M, Knave B, Lillienberg L, Westberg H, eds. X2001-Exposure assessment in epidemiology and practice. Arbete och Halsa: Stockholm: National Institute for Working Life. Vetenskaplig Skriftserie Nr. 2001;10:6-7.

35. Burstyn I, Teschke K. Studying the determinants of exposure: a review of methods. Am Ind Hyg Assoc J. 1999;60:57-72.

36. Fritschi L, Nadon L, Benke G, Lakhani R, Latreille B, Parent ME, et al. Validation of expert assessment of occupational exposure. In: Hagberg $\mathrm{M}$, Knave B, Lillienberg L, Westberg $\mathrm{H}_{t}$ eds. X2001-Exposure assessment in epidemiology and practice. Arbete och Halsa: Stockholm: National Institute for Working Life. Vetenskaplig Skriftserie Nr. 2001;10:6-7.

37. Monge $\mathrm{P}$, Wesseling $\mathrm{C}$, Engel L, Rojas $\mathrm{M}$, Zuurbier M, Keifer M, et al. Determinants of parental occupational pesticide exposure in a case-control study of childhood leukemia. (En preparación).

38. Arbuckle TE, Burnett R, Cole D, Teschke K, Dosemeci M, Bancej C, et al. Predictors of herbicide exposure in farm applicators. Int Arch Occup Environ Health. 2002;75:406-14.

39. Brouwer DH, Brouwer EJ, van Hemmen JJ. Estimation of long-term exposure to pesticides. Am J Ind Med. 1994;25:573-88.

40. Tielemans E, Heederik D, Burdorf A, Vermeulen $\mathrm{R}$, Veulememans $\mathrm{H}$, Kromhout $\mathrm{H}$, et al. Assessment of occupational exposures in a general population: comparison of different methods. Occup Environ Med. 1999;56: 145-51.

Manuscrito recibido el 13 de octubre de 2004. Aceptado para publicación, tras revisión, el 8 de abril de 2005
ABSTRACT

\section{Determining exposure history in occupational epidemiology}

Key words Occupational risk; epidemiologic methods; risk assessment.
In epidemiology, it is necessary that exposure indicators have good validity in order to obtain valid results when measuring the risks associated with occupational exposure to environmental noxious agents. However, ensuring the validity of past exposure data is no easy task.

Because there are no environmental hygiene measures or representative levels of bioindicators signaling past exposure, self-reports have been used as a source of indirect exposure data. Unfortunately, data on specific agents are commonly poor and need to be complemented with data on the determinants of exposure. The validity of self-reports improves when certain techniques, such as control lists and icons, are employed, and the quality of individual exposure data improves when secondary data on exposure and its conditioning or determining factors are incorporated.

Exposure can be determined by means of exposure matrices, assessment by experts, and exposure models, and by using a combination of primary and secondary data on exposure and its conditioning factors. Matrices contain pooled data and can thus lead to errors in classifying individual exposure and to biased risk estimates.

Assessment by experts is probably the method with the highest validity, but it can become expensive when studies are large. It is also feasible to use a formal model for assessing perceivable exposures, complemented with expert assessments whenever the results of the model appear to deviate from reality. 